\title{
The Research of PV MPPT based on RBF-BP Neural Network Optimized by GA
}

\author{
Jian Liu \\ SHENYANG JIANZHU UNIVERSITY \\ Shenyang, China \\ xxliujian@sjzu.edu.cn \\ Xiaolin $\mathrm{Xu}$ \\ SHENYANG JIANZHU UNIVERSITY \\ Shenyang, China \\ 458039912@qq.com \\ * Corresponding Author
}

\author{
Tong Li \\ SHENYANG JIANZHU UNIVERSITY \\ Shenyang, China \\ 676887576@qq.com \\ Meiyan Cao \\ li SHENYANG JIANZHU UNIVERSITY \\ Shenyang, China \\ 1099168914@qq.com
}

\begin{abstract}
In order to track nominal output power of photovoltaic (PV) battery effectively and consider the feature of non-linear output, researchers present a Radial Basis Function and Back Propagation (RBF-BP) combination neural network based on genetic algorithm (GA) optimization to use in PV maximum-power-point-tracking (MPPT).First, combination of double hidden layer RBF - BP neural network is presented by researching the output feature of PV battery. In order to predict the maximumpower-point of $\mathrm{PV}$ battery more accurately, GA is used to optimize combination neural network. Illumination and temperature which is the main factors influencing the output of PV battery are treated as input to construct the prediction model, and simulate the model through MATLAB. Simulation shows that the system has advantages which increase the accuracy and efficiency of tracking the output maximum-power-point-tracking of $P V$ battery effectively of high tracking accuracy, high speed rate and little iteration.
\end{abstract}

Keyword- Photovoltaic (PV) battery; Maximum-powerpoint-tracking (MPPT); genetic algorithm (GA); Radial Basis Function and Back Propagation (RBF-BP); MATLAB simulation

\section{INTRODUCTION}

As traditional energy consumption brought more serious environmental problems coupled with the development of modern society, power quality requirements are high ${ }^{[1]}$. Solar energy as a new, environmentally friendly, renewable energy has got more and more attention ${ }^{[2]}$. PV power generation which is directly converted solar energy into electrical energy has quickly become a renewable energy technology, which has the potential for large-scale development in the future and it may become an important alternative energy source ${ }^{[3]}$. In order to improve the efficiency of PV power generation, maximum power point tracking for PV power generation is particularly necessary. In recent years, domestic and foreign scholars have proposed a variety of maximum power point tracking algorithm, such as perturbation and observation control ${ }^{[4]}$, conductance increment method ${ }^{[5]}$ and neural network method $^{[5,6]}$ and so on. Though having good results in a faster rate of change of the light situation, perturbation and observation control require higher precision of the sensor and long running time. The disadvantage of conductance increment method is the step length is fixing. When the step length is too small, the prediction result of $\mathrm{PV}$ is easy to remain in the low power output region. When the step length is too large, the measurement results will significantly oscillate. In contrast artificial neural network method not only has a strong ability of nonlinear function approximation, but also does not require the physical parameters of the PV battery in the establishment of the model. $\mathrm{BP}{ }^{[6]}$ and $\mathrm{RBF}^{[7]}$ are widely used around the existing artificial neural network. BP neural network has strong self-learning ability, but the network may misleading by partial sample resulting in the deviation of learning outcomes; RBF neural network has faster convergence rate, but it is lack of generalization ability. This paper proposed a combination of double hidden layer RBF- BP neural network with both advantages, and optimized this neural network by PV. Simulation and analysis through the MATLAB, combination neural network based on PV optimization increase the speed and accuracy of prediction is verified.

\section{PV CELL MODELS AND CHARACTERISTICS}

\section{A. The mathematical model of PV cells}

According to the theory of electronics, equivalent circuit of solar battery is shown as Figure $1_{f}^{[8]}$.

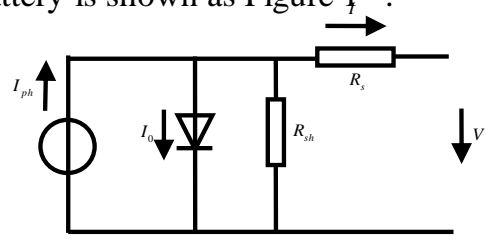

Figure 1. Solar battery equivalent circuit 
The equivalent mathematical description of PV cells as follows:

$$
I=I_{p h}-I_{0}\left\{\exp \left[\frac{q\left(V+I R_{s}\right)}{A K T}\right]-1\right\}-\frac{V+I R_{s}}{R_{s h}}
$$

Where, I is the battery output current and V is the output voltage; ${ }^{I}{ }_{p h}$ is Photo production current and $I_{0}$ is reverse saturation current inside the battery equivalent diode $\mathrm{p}-\mathrm{n}$ junction ; $\mathrm{q}$ is electronic charge which is $1.6 \times 10^{-19} \mathrm{C}$;A is ideal factor of diode $\mathrm{PN}$ junction; $\mathrm{K}$ is The boltzmann constant which is $1.38 \times 10^{-23} \mathrm{~J} / K$ and $\mathrm{T}$ is working temperature; $\mathrm{R}$ is series resistance and $R_{s h}$ is parallel resistance.

\section{B. The output characteristics of PV cells}

Since the output of PV battery is affected by multiple factors such as temperature, light intensity, and its output characteristic presents obvious nonlinear. This paper mainly focuses on considering the influence of the temperature and light intensity of PV panels for PV cells output.

According to the mathematical model of the PV cells, this paper selects Solarex MSX60 $60 \mathrm{w}$ battery template MATLAB simulation of electrical parameters ( $V_{O C}=21 \mathrm{~V}, I_{s c}=3.74 \mathrm{~A}, V_{m}=17.1 \mathrm{~V}, I_{m}=3.5 \mathrm{~A}, P_{m}=59.9 \mathrm{~W}$ ) then researchers got the $\mathrm{P}-\mathrm{V}$ curves of the PV cells as Figure 2 and Figure 3

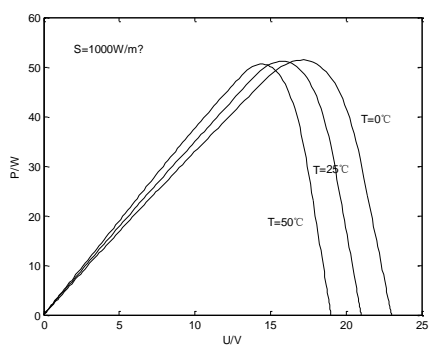

Figure 2. $\mathrm{P}-\mathrm{V}$ curves when $\mathrm{T}$ change and $\mathrm{S}$ is constant

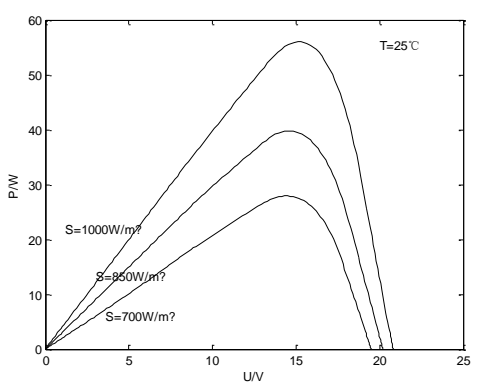

Figure 3. $\mathrm{P}-\mathrm{V}$ curves when $\mathrm{S}$ change and $\mathrm{T}$ is constant

Through analysis Figure 2 shows the output of the PV cells as the typical nonlinear.

Under the condition of light intensity is constant, the temperature is higher, the peak value of the output power is smaller. When under the condition of constant temperature, light intensity is greater, the peak of the output power is greater.
The simulation results shows that the output of the PV cell is a typical nonlinear, and under different working conditions, the output power with the increase of the output voltage to the change trend of nonlinear first becomes big and then small ${ }^{[9]}$. Therefore, in order to improve the output efficiency of PV cells, it is particularly necessary for accurate fast tracking of maximum power point of PV cells.

\section{THE RESEARCH OF MPPT METHODS BASED ON RBF-BP NEURAL NETWORK OPTIMIZED BY GA}

\section{A. RBF-BP neural network}

This paper present a RBF-BP neural network which is combined with advantages of strong learning ability, high fitness, converging fast of RBF neural network and high group classification performance ,building a combination of double hidden layer RBF- BP neural network combined with high fitness, converging fast and High precision. RBF-BP neural network structure is shown as Figure 4.

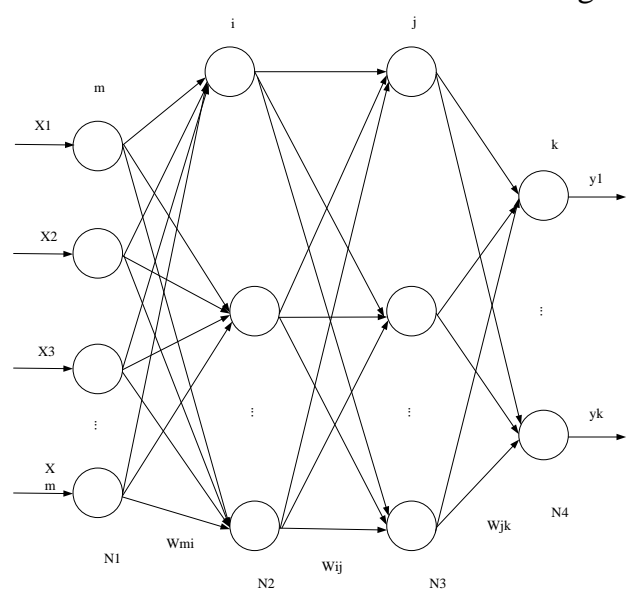

Figure 4. RBF-BP network structure

As shown in Figure 4, Sample data is preferentially trained by RBF neural network and the training result as input of the BP neural subnet. Network's initial weights and threshold value are random numbers between $(0$, 1).When the training accuracy did not reach the goal, the neural network can reverse change network weights and threshold until the training results meet the accuracy requirements. When the precision of the training results reach the expected value, end the training .

\section{B. RBF-BP neural network with GA optimized}

Due to the combination of RBF-BP neural network training data when the initial weights and thresholds for the random number between $(0,1)$, in order to meet the requirements of the accuracy of the anticipated target, RBF-BP neural network to reverse changes according to the actual situation of the study result weights and thresholds, the process will undoubtedly reduce the efficiency of the whole network prediction.

In order to solve the initial weights and threshold of uncertainty on the efficiency of the combination of RBF$\mathrm{BP}$ neural network prediction, this paper present using the combination of GA for RBF-BP neural network for further optimization as Figure 5. 


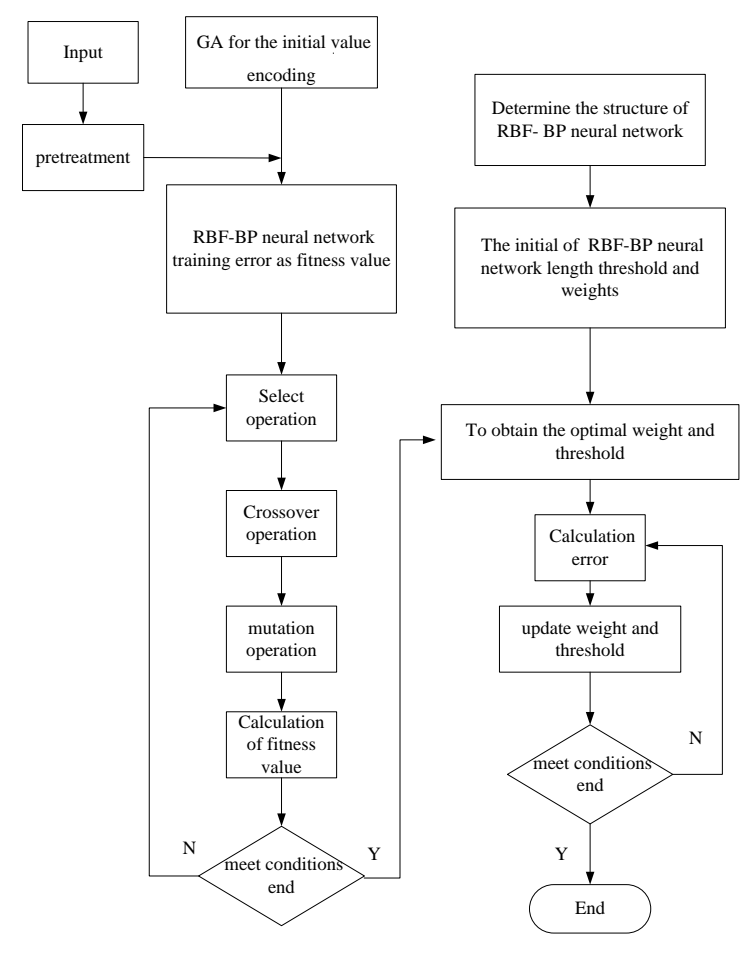

Figure 5. RBF-BP network process with GA optimized

RBF-BP neural network with the GA optimized is divided into RBF-BP neural network structural and GA optimization from Figure 5.Each individual in the sample population contains the weight and threshold of the neural network..GA optimizing parts find a fitness which is the absolute value of the error between the output and the desired output as a benchmark,using GA on the sample data individual species selection, crossover and mutation operation to find the individual corresponding to the optimal fitness, and determine the optimal initial threshold and weight is assigned to the neural network .

\section{The research of the neural network optimized of $P V$ $M P P T$}

The maximum output power of PV battery is affected by multiple factors such as temperature, sunshine intensity.According to the actual situation in this paper, researchers focus on considering the temperature of the PV panels,light intensity and the output voltage of maximum power point as the main input and output parameters of neural network training .RBF-BP neural combined with input layer, hidden layer and output layer.Input layer: without considering the complex weather conditions and power board under the condition of uneven illumination, for PV maximum power prediction of main consideration PV cells measured when working temperature and light intensity as two input nodes .Hidden layer:RBF neural network is adopted in combination of RBF-BP neural network hidden layer and its output as the input of the BP neural network, thus set a RBF neural network node number is 9, BP neural network node number is 5 .Output layer: in order to reflects the maximum power output of PV battery point effective, researchers select output voltage of maximum power point of PV cell to be only .

In order to improve the efficiency and accuracy of the combination of RBF-BP neural network to PV MPPT, avoid uncertainty due to network the initial weights and threshold of PV forecasting, this design uses GA to optimize the initial threshold and weight of RBF-BP neural network methods:

1) Researchers put part of the training sample data into RBF-BP neural network, and get the error between the actual output and the expected output for fitness value of the GA.

2) According to the number of input and output parameters of RBF-BP neural network, researchers determine the number of the threshold and the weights. Then researchers determine the length of the individuals of GA

3) Optimization of genetic algorithm for training data, the training sample of each individual contains the RBF-BP weight value and threshold value of BP neural network, individual coding method for real number encoding. Each individual is a real number. Individual fitness value of the individual is calculated by GA fitness function calculation .Then:

(1) Select operation

Researchers choose roulette wheel method,Such as Formula (2)

$$
\left\{\begin{array}{l}
f_{n}=k / F_{n} \\
p_{n}=\frac{f_{n}}{\sum_{i=1}^{M} f_{n}}
\end{array}\right.
$$

$p_{n}$, choice probability of every individual $\mathrm{n}$ in $\mathrm{A}$ population; $f_{n}$, The reciprocal of fitness of individual $\mathrm{n} ; \mathrm{k}$, coefficient; M, Number of individual species

(2) Crossover operation

In this paper, researchers use the real crossing method, crossover operation of the first $\mathrm{K}$ chromosome $X_{k}$ and $\mathrm{A}$ chromosome $X_{1}$ in $\mathrm{j}$, as shown in Formula (3):

$$
\left.\begin{array}{l}
X_{k j}=X_{k j}(1-b)+X_{l j} b \\
X_{l j}=X_{l j}(1-b)+X_{k j} b
\end{array}\right\}
$$

B A random number between the $[0,1]$.

\section{(3) Mutation operation}

Researchers select the $\mathrm{j}$ genes of the individuals i $X_{i j}$ to mutation, such as Formula (4):

$$
X_{i j}= \begin{cases}X_{i j}+\left(X_{i j}-X_{\max }\right) * f(g) & a>0.5 \\ X_{i j}+\left(X_{\min }-X_{i j}\right) * f(g) & a \leq 0.5\end{cases}
$$

$X_{\max }$,upper limit of gene $X_{i j} ; X_{\text {min }}$,lower limit of gene $X_{i j} ; f(g)=r\left(1-g / G_{\max }\right)^{2} ;$ r,a random number; $\mathrm{g}$, current iteration number; $G_{\max }$ maximum evolutionary times; a, a random number between the $[0,1]$.

The initial weights and thresholds which are best adaptive individuals obtained by GA selection, crossover and mutation operation of RBF-BP neural network are 
assigned. Then researchers predict the maximum output power of PV cells by training RBF-BP neural network.

\section{SIMULATION RESULTS AND ANALYSIS}

Researchers achieve the training and the iterative process of RBF-BP neural network optimized with GA by MATLAB R2009a. There is a total of 144 sets of experimental data of the $5 \mathrm{~min}$ of the PV panels, the temperature and the intensity of the light intensity in the day during $6 \mathrm{~h}$ to $18 \mathrm{~h}$ from the reference ${ }^{[10]}$. Test data is 24 groups of the power plate temperature and light intensity collected of every $30 \mathrm{~min}$ acquisition from $6 \mathrm{~h}$ to $18 \mathrm{~h}$ in next day.

Programming and setting the combination of RBF-BP neural network with GA optimized through MATLAB , combination of RBF-BP neural network training uses trainlm function, hidden layer of $\mathrm{RBF}$ and $\mathrm{BP}$ are adopted radbas function and Tansig function as the transfer function. Transfer function of the output layer uses purelin function. The evolution iteration number of GA is 100; the crossover probability is 0.4 . The mutation probability is 0.1.The maximum number of training times of the network is set to 1000 times. The expected error is set to 0.00001 and the learning rate is set to 0.01 . The process of generating the individual which suit optimal fitness from the sample data through GA selection, crossover and mutation as Figure 6

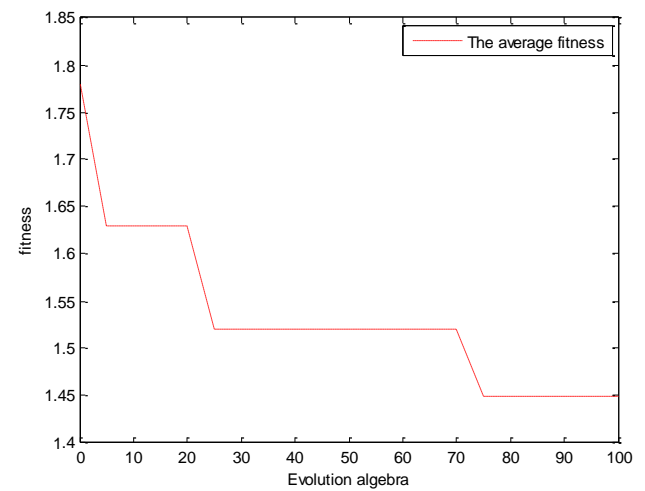

Figure 6 . The process of generating the individual which suit optimal fitness

The simulation results of Figure 7 (a),(b) are training error curve of the RBF-BP neural network with GA optimized and combination of RBF-BP neural network .Obviously, though lack of training data and sample data, under the same accuracy requirement, combination of RBF-BP neural network need training 44 epochs and combination of RBF-BP neural network with GA optimized which improve the efficiency need only 39 epochs.

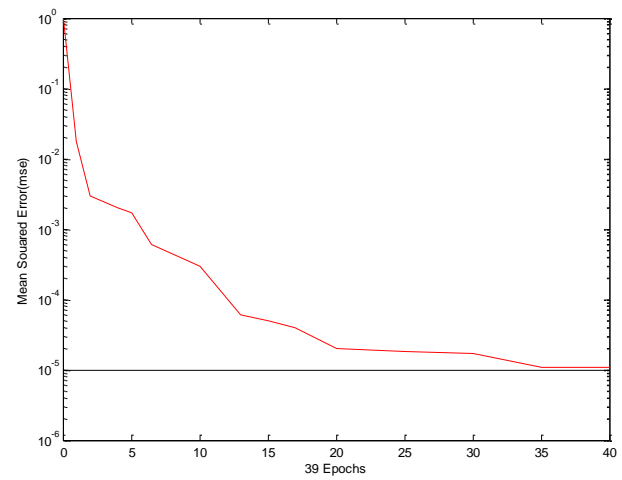

(a) Training error curve of RBF-BP neural network with GA optimized

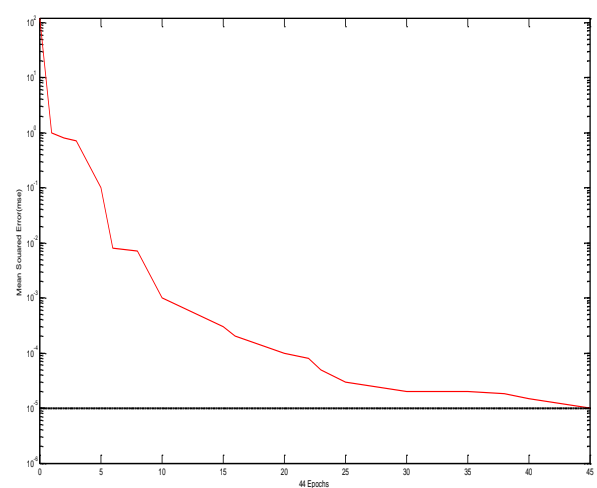

(b) Training error curve of RBF-BP neural network

Figure 7 . Training error curve of Neural network

Square error of combination of RBF-BP neural network with GA optimized and RBF-BP neural network were $9.85791 \times 10^{-5}$ and $1.3573 \times 10^{-4}$ from the MATLAB MSE function of Figure 7(a) and (b). By contrast, prediction accuracy and efficiency of combination of RBF-BP with GA optimized neural network for maximum power output of PV battery point has improved obviously.

In order to reflect the accuracy of the combination of RBF-BP neural network with GA optimized intuitively, Figure 7 shows 24 set of the actual error distribution of test sample point. The error of the maximum power point voltage of values by network predicted is between 0.02 0.02 from Figure8. It is showed that the combination of RBF-BP neural network with GA optimized is used to track the maximum power point of $P$ $\mathrm{V}$ cells with high accuracy. 


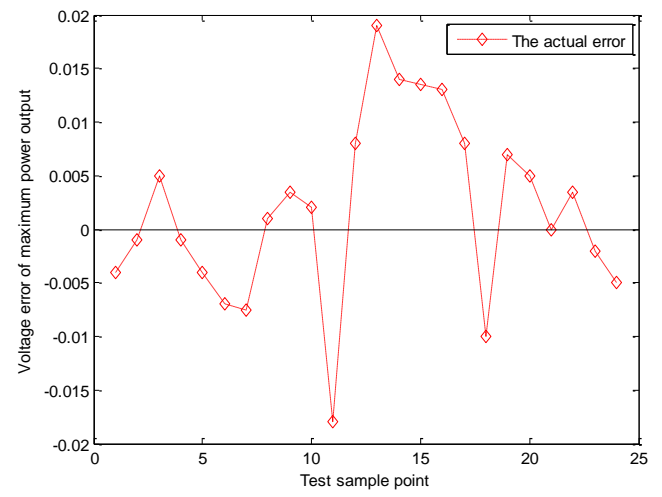

Figure 8 . Test error Figure

\section{V.CONCLUSIONS}

PV array operating temperature and optical light intensity are selected as input of RBF-BP combined neural network without considering the complex weather conditions in prediction model of this paper and make optimization on combined neural network using GA at the same time. Therefore, tracking speed and accuracy of MPPT of PV cells are improved. Results of simulation also show that optimized RBF-BP combined neural network by GA is better than no optimized combined neural network both on the number of iterations and the accuracy of prediction. Therefore, the reliability of the RBF-BP combined neural network using GA optimized is proved and the problem of MPPT of PV array is effectively solved.

\section{REFERENCE}

[1] Fang liang-fei.Research on the control strategy of photovoltaic energy storage [D]. HeFei University of Technology,2010.

[2] Zhao zheng-ming,Liu jian-zheng,Sun xiao-ying.Solar photovoltaic power generation and its application[M].Beijing:Science Press,2005.

[3] Zhu yan-wei,Shi xin-chun,Dan yang-qing.et al.Application of the particle swarm optimization algorithm in multi peak maximum power point tracking of photovoltaic array $[\mathrm{J}][\mathrm{J}]$. Chinese Journal of electrical engineering,2012,32(4):42-48.

[4] Femia N, Petrone G, Spagnuolo G, et al. Optimization of perturb and observe maximum power point tracking method[J].IEEE Transactions on Power Electronics,2005,20(4):963-973.

[5] Yusof Y, Sayuti S H,A bdul Latif M, et al. Modeling and simulation of maximum power point tracker for $\mathrm{PV}$ system[C].Proceedings of National Power and Energy Conference. Kuala Lumpur, Malaysia,2004:88-93.

[6] Liang xing-yan,Zhang wei.Research of MPPT based on BP neural network for photovoltaic power generation system [J] manufacturing automation,2012, (24):112-114,130.

[7] Li tan,Dong hai-ying, Yang lei.Photovoltaic power generation technology of RBF neural network based on ant colony algorithm MPPT[J]. neural network,2014, 38(9):1644-1646.

[8] Zhang jian-po,Zhang hong-yan,Wang tao,et al.Simulation Research on the maximum power tracking algorithm in photovoltaic system [J]. computer simulation,2010,27(1):266-270.

[9] Fu wang,Luo shi-wu,Qing zhi-ming. Research on the mathematical model of PV array under the condition of partial shading [J]. computer simulation 2013,30(7):120-124.

[10] Guo liang.Maximum power point tracking of photovoltaic system based on particle swarm optimization BP neural network [D] Southwest Jiao Tong University,2011 